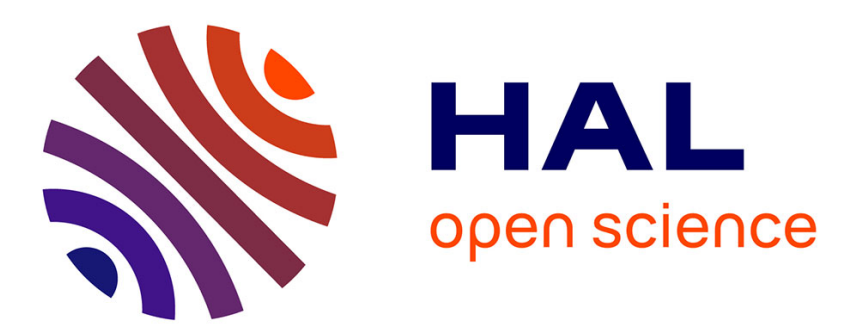

\title{
Thermoviscoelastic analysis of concrete creep at mesoscale
}

B. Bary, C. Bourcier, T. Helfer

\section{To cite this version:}

B. Bary, C. Bourcier, T. Helfer. Thermoviscoelastic analysis of concrete creep at mesoscale. CONSEC 2016 - 8th International conference on concrete under severe conditions-environment and loading, Sep 2016, Lecco, Italy. cea-02509682

\section{HAL Id: cea-02509682 https://hal-cea.archives-ouvertes.fr/cea-02509682}

Submitted on 17 Mar 2020

HAL is a multi-disciplinary open access archive for the deposit and dissemination of scientific research documents, whether they are published or not. The documents may come from teaching and research institutions in France or abroad, or from public or private research centers.
L'archive ouverte pluridisciplinaire HAL, est destinée au dépôt et à la diffusion de documents scientifiques de niveau recherche, publiés ou non, émanant des établissements d'enseignement et de recherche français ou étrangers, des laboratoires publics ou privés. 


\title{
Thermoviscoelastic analysis of concrete creep at mesoscale
}

\author{
Benoit Bary ${ }^{1, a^{*}}$, Christophe Bourcier ${ }^{2, b}$ and Thomas Helfer ${ }^{3, c}$ \\ ${ }^{1}$ CEA, Laboratoire d'Etude du Comportement des Bétons et des Argiles, Bât. 158, F-91191 Gif-sur- \\ Yvette, France. \\ ${ }^{2}$ CEA, Laboratoire de Génie Logiciel et de Simulation, Bât. 454, F-91191 Gif/Yvette, France. \\ ${ }^{3} \mathrm{CEA}$, Laboratoire de Simulation du comportement des Combustibles, Bât. 151, 13108 St Paul lez \\ Durance.
}

abenoit.bary@cea.fr, bchristophe.bourcier@cea.fr, ${ }^{\mathrm{c}}$ thomas.helfer@cea.fr

Keywords: thermoviscoelasticity, 3D simulations, homogenization, Interfacial Transition Zone, Linear Spring Model.

\begin{abstract}
Concrete is a heterogeneous material made up at mesoscale of linear elastic aggregates distributed in a mortar matrix whose behavior is time and temperature dependent. The Interfacial Transition Zone (ITZ) between aggregates and matrix also influences the overall behavior. We investigate here analytically and numerically by means of 3D simulations the creep behavior of concrete and mortar subjected to moderate temperatures at mesoscale. The numerical specimens consist in unstructured periodic meshes of polyhedral aggregates with various size and shapes randomly distributed in a box. Specific interface finite elements are introduced between aggregates and matrix to model the ITZ. Both matrix and ITZ are considered as linear thermoviscoelastic materials. Averaged stresses and strains in the matrix and aggregate phases are compared to analytical estimations obtained with classical mean-field approximation schemes applied in the Laplace-Carson space, where the ITZ are introduced via imperfect interfaces modelled with the Linear Spring Model (LSM). The effects of ITZ thickness, aggregate shape and temperature are then studied to evaluate their respective influence on mortar and concrete creep behavior.
\end{abstract}

\section{Introduction}

Concrete may be considered as a heterogeneous material made up at the mesoscale of linear elastic aggregates distributed in a mortar matrix whose behavior is time-dependent. Besides, the presence of an ITZ between the aggregates and the matrix is known to also influence the overall behavior (see e.g. $[1,2]$ ). In the nuclear context, it is of particular importance to precisely estimate the long-term creep and induced development of cracks during the structure service life; a correct characterization of the respective role and impact of both phases and ITZ regarding the creep strains is then valuable. It is indeed well-known that initiation and propagation of cracks are strongly related to the local stresses and strains states as well as their history.

In this paper we investigate analytically and numerically at mesoscale the creep behavior of concrete regarded as a heterogeneous material with a matrix/inclusion morphology, and subjected to moderate temperature increases. The simulations are carried out on 3D concrete specimens and the computations are performed in the finite element code Cast3M (www-cast3m.cea.fr). The matrix and ITZ are considered as linear viscoelastic materials ruled by generalized Maxwell models. Specific interface elements are introduced between the aggregates and the matrix to model the ITZ. Note that to simplify the aggregates do not overlap each other, such that no percolation of the ITZ phase occurs.

One purpose of the study is to analyze the overall and intra-phase response of the numerical specimens when subjected to classical creep loadings and moderate heating. In particular, the evolutions of averaged stresses and strains in the matrix and aggregate phases will be reported and compared to analytical estimations obtained with classical mean-field approximation schemes 
applied in the Laplace-Carson (LC) space. The effects of the ITZ will be accounted for via interfaces modelled with the linear-spring model (LSM) (see e.g. [3]). The influence of the ITZ thickness on the overall response of the specimens will also be studied.

\section{Modelling}

Viscoelasticity. As mentioned in the introduction, we are concerned with the analysis of the viscoelastic behavior (see e.g. [4,5] for a comprehensive description of linear viscoelasticity) of concrete with a special focus on the effects of the ITZ. The behavior of the matrix material is assumed to be linear viscoelastic, with bulk $k^{m}(t)$ and shear $\mu^{m}(t)$ moduli ruled separately by a generalized Maxwell model with 3 viscoelastic chains (the element labeled as 0 contains only a spring element) as:

$$
k^{m}(t)=k_{0}^{m}+\sum_{i=1}^{3} k_{i}^{m} e^{-t / \tau_{i}^{m}}, \mu^{m}(t)=\mu_{0}^{m}+\sum_{i=1}^{3} \mu_{i}^{m} e^{-t / \tau_{i}^{m}}
$$

in which $k_{i}^{m}$ and $\mu_{i}^{m}$ with $i \in\{0,3\}$ are the elastic moduli of the Maxwell chains and $\tau_{j}^{m}$ are their relaxation times. It is well-known that in the case of linear viscoelasticity the time-dependent problem may be equivalently reformulated as a linear elastic problem in the LC space, allowing to applying classical upscaling techniques (see e.g. [6-8]). The macroscopic behavior may take the form of a linear viscoelastic material:

$$
\boldsymbol{\sigma}(t)=3 \int_{0^{-}}^{t} k^{h o m}(t-\tau) \frac{d \epsilon}{d \tau} d \tau \mathbf{1}+2 \int_{0^{-}}^{t} \mu^{h o m}(t-\tau) \frac{d \boldsymbol{e}}{d \tau} d \tau
$$

with $\boldsymbol{\sigma}(t), \epsilon=1 / 3 \operatorname{tr}(\boldsymbol{\varepsilon})$ and $\boldsymbol{e}$ the macroscopic stress tensor, bulk and deviatoric part of the strain tensor $\boldsymbol{\varepsilon}$, respectively; $k^{\text {hom }}$ and $\mu^{\text {hom }}$ are the homogenized bulk and shear moduli, which are expressed in the form of Prony series due to the collocation method employed to determine the solution of the inverse LC transform problem. It is noteworthy that in [9] exact or semi-analytical solutions of the inversion problem are also derived in the form of Eqs. (1-2) in the particular case of the Mori-Tanaka scheme and spherical particles.

Interface modelling. The behavior of the interface between the aggregates and the matrix is also assumed to be ruled by generalized Maxwell models. As in concrete the mechanical properties of the ITZ are known to be more compliant than those of the mortar matrix and aggregates, we apply the LSM in the LC space to model the interface effects. The interface conditions for the LSM are expressed as (e.g., $[3,10])$ :

$$
[\boldsymbol{\sigma}] \cdot \boldsymbol{n}=0, \mathbf{k} .[\boldsymbol{u}]=\boldsymbol{\sigma} \cdot \boldsymbol{n}
$$

where $\boldsymbol{n}, \mathbf{k}, \boldsymbol{u}$ are the unit normal vector to the interface, the second order tensor characterizing the elastic parameters in the LC space, and the displacement, respectively; [·] denotes the jump across the interface of the corresponding quantities. In the case where the interface is thin and compliant with respect to the inclusion, i.e. $h \ll R_{i}, E_{c} \ll E_{i}$ and $\mu_{c} \ll \mu_{i}$ with $h$ the thickness of the interphase, $R_{i}$ the radius of the spherical inclusion, $E_{c}$ and $\mu_{c}$ the Young and shear moduli of the interphase, $E_{i}$ and $\mu_{i}$ the Young and shear moduli of the inclusion, the tensor $\mathbf{k}$ can be expressed as $\mathbf{k}=k_{n} \boldsymbol{n} \otimes \boldsymbol{n}+k_{t} \boldsymbol{s} \otimes \boldsymbol{s}+k_{t} \boldsymbol{t} \otimes \boldsymbol{t}$ with:

$$
k_{n}=\frac{2 \mu_{c}\left(1-v_{c}\right)}{h\left(1-2 v_{c}\right)}, k_{t}=\frac{\mu_{c}}{h}
$$


in which $\boldsymbol{s}$ and $\boldsymbol{t}$ are the two orthogonal unit vectors in the tangent plane of the interface and $v_{c}$ is the Poisson ratio of the interphase. In the following, we will retain this model for characterizing the properties of the interfaces in the FE simulations and in the analytical formulation.

Analytical homogenization with interfaces. We propose to estimate the overall properties of the heterogeneous material by making use and extending the approach due to [3], based on a replacement procedure (see Fig. 1). In this approach, the elastic composite inclusions composed of the spherical aggregates and their surrounding interfaces are replaced by equivalent homogeneous spherical inclusions. The expressions of the moduli for these equivalent particles depend on both inclusion and interphase properties; they take the following form (see [3] for details):

$$
k_{e q}=\frac{m_{r} k_{i} \mu_{m}}{3 k_{i}+m_{r} \mu_{m}}, \mu_{e q}=\frac{\mu_{i}\left(24 M m_{\theta}+m_{r}\left(16 M+m_{\theta} N\right)\right)}{80 g_{3} M+4 g_{3} m_{\theta}\left(10\left(7-v_{i}\right)+M\right)+m_{r}\left(2 g_{3}\left(140-80 v_{i}+3 M\right)+m_{\theta} N\right)}
$$

with $M=g_{3}\left(7+5 v_{i}\right), \quad N=5\left(28-40 v_{i}+M\right), \quad g_{3}=\mu_{i} / \mu_{m}, \quad m_{r}=k_{n} R_{i} / \mu_{m} \quad$ and $m_{\theta}=$ $k_{t} R_{i} / \mu_{m}$. In these relations the indexes $m$ and $i$ relate to the matrix and inclusion phases, respectively. Since these formulas are established in an elastic framework, they are applied here in the LC space. Once the composite inclusions are substituted by equivalent homogeneous ones, upscaling schemes may be applied to obtain the overall properties of the concrete material. In the applications, we will make use essentially of the generalized self-consistent scheme (GSCS) which is known to give satisfying estimations even for relatively large inclusion volume fractions (see e.g. $[11,12])$.

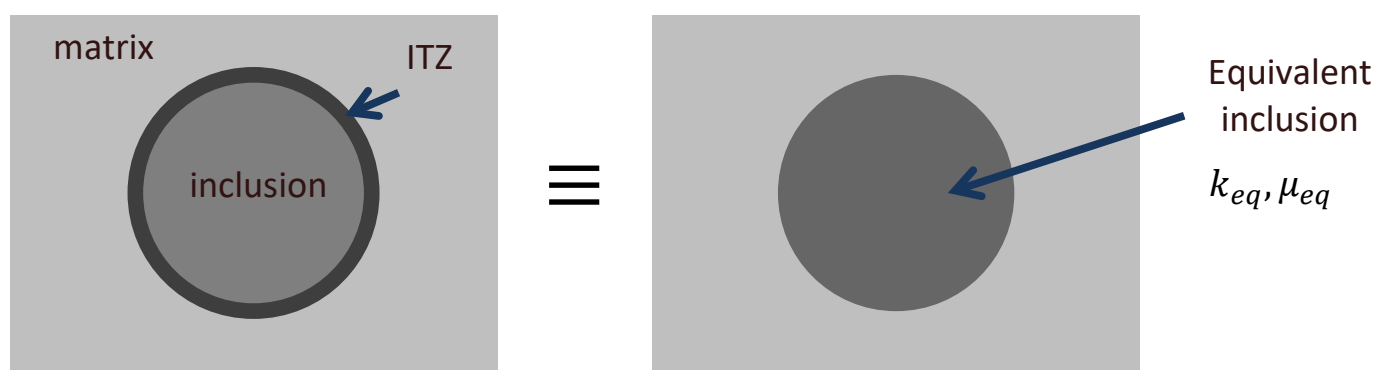

Fig. 1. Description of the replacement procedure [3].

Construction of the mesostructures. The procedure for generating the numerical samples is detailed in [13,14]. The open-source python library Combs [13] based on the Computer-Aided Design code Salome (www.salome-platform.org) is used to generate both geometry and meshes of the mesostructures. The geometries considered in this paper are obtained by randomly distributing in a box polyhedral aggregates of various sizes and shapes obtained by a classical Voronoi space decomposition [14,15]. To limit the FE computation time and the placement procedure, the choice of 4627 coarse aggregates has been retained, for a total volume fraction of 0.50 . Their number and volume are defined to match the ones of the corresponding spheres assembly according to the sieve curve described in [14]. The total number of aggregates in the samples is greater than the prescribed one since the aggregates overlapping the surfaces of the mesostructures are cut and the remaining parts are placed on the opposite faces to ensure periodicity. Fig. 2 presents the mesh of a sample realization with isotropic particles. As mentioned in the introduction, the aggregates do not intersect each other by construction (note that this is also the case for the analytical estimations, and on that point both microstructure representations coincide), so that no ITZ percolation is considered. This aspect would however deserve further investigations in the future. 


\section{Extension to linear thermoviscoelasticity}

We extend in this section the formulation presented above to the case of linear thermoviscoelasticity. The effects of temperature have to be introduced in the model through different aspects. One relates to the viscoelastic behavior of the matrix phase, which is known to be strongly affected by temperature (see e.g. [16,17]. A simple approach to account for this effect is the time shift (or reduced time) method which consists in replacing the variable time $t$ by an equivalent time variable $t_{e}(t)$ defined as (see e.g. [16]):

$$
t_{e}(t)=\int_{0}^{t} \exp \left[-\frac{Q}{R}\left(\frac{1}{T(\tau)}-\frac{1}{T_{0}}\right)\right] d \tau
$$

where $Q$ is an activation energy, $R$ is the ideal gas constant, $T_{0}$ is a reference temperature and $T$ is the current temperature. For the applications, the ratio $Q / R$ is set to $6200 \mathrm{~K}$, which is close to values found in the literature for concrete materials (see e.g. [9,17]).

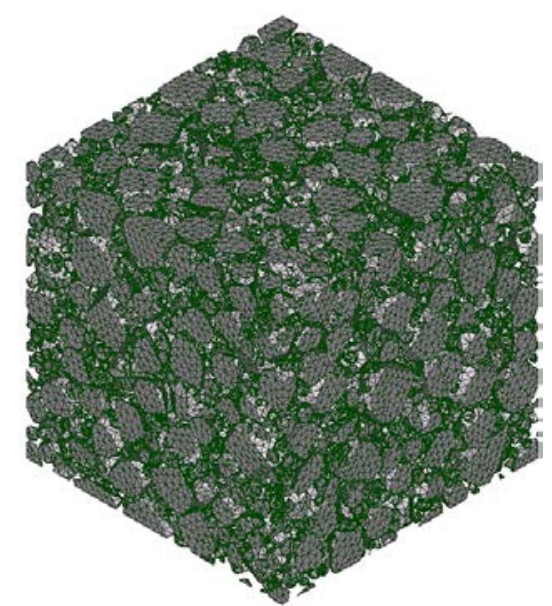

Fig. 2. Mesh of a sample with isotropic aggregates.

Another aspect to consider regarding temperature effects is the dilatation of the material phases. Here the coefficients of thermal expansion (CTE) of aggregates $\alpha_{g}$ and matrix $\alpha_{m}$ are assumed constant and equal to $1.1 \times 10^{-5}$ and $1.5 \times 10^{-5} \mathrm{~K}^{-1}$, respectively, corresponding to average values for sand and cement paste from [18,19]. The CTE of the ITZ phase $\alpha_{c}$ is estimated from the matrix one by describing it as a composite constituted by the matrix material with additional porosity. The next step of the modelling is to calculate the effective CTE $\alpha_{\text {eff }}$ of the 3-phase material composed of spherical aggregates surrounded by an ITZ layer and immersed in the matrix. We make use here of the developments of [20] to derive $\alpha_{e f f}$ in the case of thin interfaces with elastic mechanical parameters much lower than those of the inclusions, leading to the explicit expression:

$$
\frac{\alpha_{e f f}-\phi_{g} \alpha_{g}-\phi_{m} \alpha_{m}-\frac{\phi_{g} \gamma}{R_{i}}}{\frac{1}{k_{e f f}}-\frac{\phi_{g}}{k_{g}}-\frac{\phi_{m}}{k_{m}}-\frac{3}{k_{n} R_{i}}}=\frac{\alpha_{g}-\alpha_{m}+\frac{\gamma}{R_{i}}}{\frac{1}{k_{g}}-\frac{1}{k_{m}}+\frac{3}{k_{n} R_{i}}}
$$

where the subscripts $m, c$ and $g$ designate again the matrix, interphase and aggregate, respectively; $\phi_{i}$ is the volume fraction of phase $i$; $k_{n}$ and $k_{t}$ are defined in Eqs. (4). Finally, $\gamma$ is the normal thermoelastic parameter of the interface defined by $[\boldsymbol{u}] . \boldsymbol{n}=\gamma \Delta T$ with $\Delta T$ the increment of temperature. The parameter $\gamma$ defines the response of the interfaces in terms of normal displacement jump when subjected to a temperature variation. Again, we refer here on the results derived by [20] to express $\gamma$ as a function of the thermoelastic properties of interphase and aggregates, and $h$, as:

$$
\gamma=\frac{\alpha_{c}\left(1+v_{c}\right)-2 \alpha_{g} v_{c}}{1-v_{c}} h
$$


It should be recalled that all the above expressions are defined in the LC space, for their extension in the linear thermoviscoelastic case. For the FE, the resulting thermoviscoelastic behaviors of both interface and matrix (i.e. volume finite elements) have been implemented via the code generator MFront (www.tfel.sourceforge.net, [21]), and the general thermo-mechanics formulation of Cast3M has been applied.

\section{Results}

Creep results. We first present results obtained with the mesostructure with isotropic Voronoï aggregates in the case of a classical creep loading. The simulations are performed with homogeneous stresses boundary conditions (BC). The analytical estimations are derived with the replacement procedure and the GSCS with 2 phases. Fig. 3 left presents the evolutions of the creep longitudinal and transversal strains obtained numerically by FE (symbols) and analytically (lines) for the different interface thicknesses of $h=1,25,50,100$ and $250 \mu \mathrm{m}$. We observe as expected that the magnitude of strains progressively increases for higher values of thickness. The differences are small between $h=1$ and $25 \mu \mathrm{m}$, and are about 6, 14 and 33\% between $h=1$ and 50, 100 and $250 \mu \mathrm{m}$, respectively. Note that the results with no interface are omitted as they are superposed with the $h=1$ thickness ones. One can conclude that the presence of ITZ, whose typical thickness is around 20-25 $\mu \mathrm{m}$ in concrete at mesoscale (i.e. considering the matrix as mortar), is not significant regarding the estimation of macroscopic response of the material. By contrast, for mortars which correspond approximately to the case of a thickness of $100 \mu \mathrm{m}$ with respect to the aggregate sizes, they appear influential and should not be neglected.
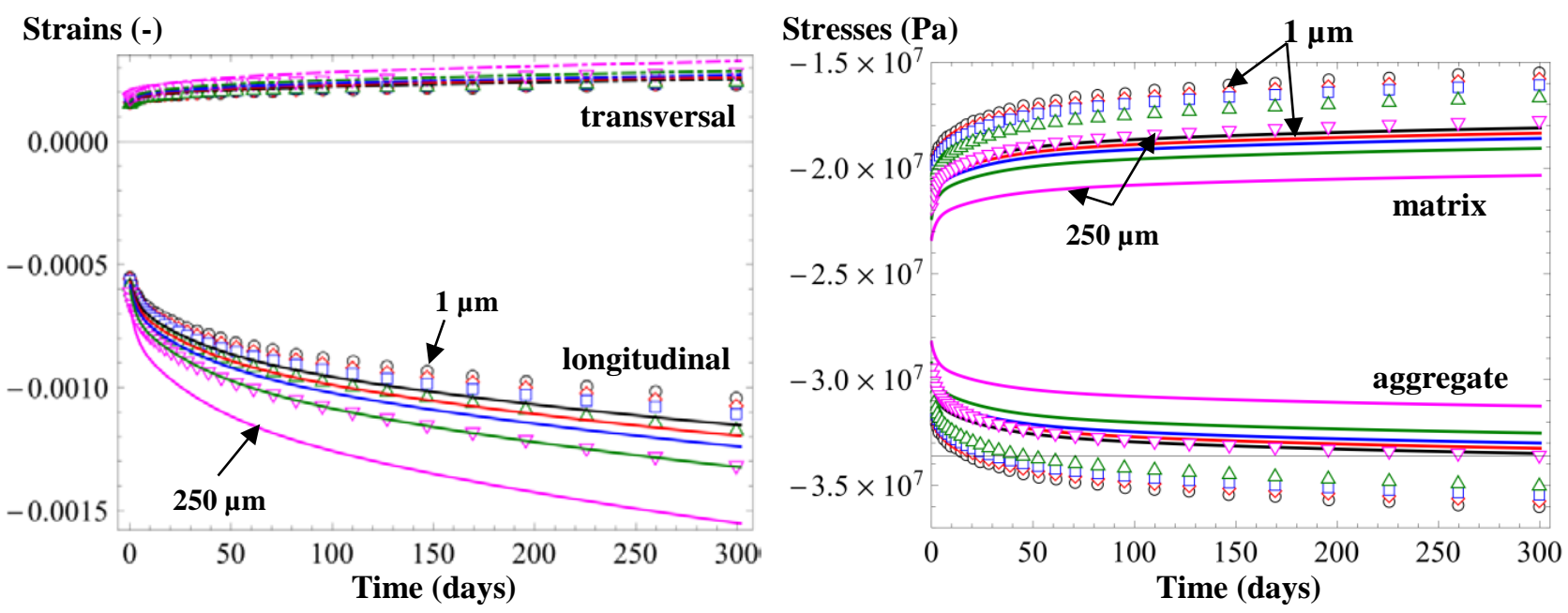

Fig. 3. Longitudinal and transversal creep strains obtained numerically (symbols) and analytically (lines) for interface thicknesses of 1, 25, 50, 100 and $250 \mu \mathrm{m}$ (left). Corresponding average stresses in the matrix and aggregate phase for the same interface thicknesses (right).

We also observe that the strains estimated by the model overestimate in magnitude the numerical ones for all interface thicknesses. Several causes may explain these discrepancies. The first and most important one lies in the inclusion shape considered, spherical for the model and polyhedrons for the FE; another is due to the inherent use of the GSCS which does not provide exact results (with respect to the microstructure described here). Biases introduced by the insufficiently fine meshes and the considered BC are also well-known sources of inaccuracies. Fig. 3 right shows the evolutions of average stresses in the aggregates and matrix in the direction of creep loading, obtained numerically (symbols) and analytically (lines) for the different interface thicknesses. This Fig. indicates that an increase of the interface thickness tends to reduce the magnitude of the stress in the aggregates and to augment it in the matrix. Again, the model provides estimations relatively close to the FE simulations. 
Thermoviscoelastic results. We present in this subsection illustrative simulation results when the only external loading is a temperature increase of $40^{\circ} \mathrm{C}$. The simulations are performed with homogeneous stresses BC. The analytical estimations are derived with the replacement procedure and the GSCS with 2 phases. Fig. 4 (left) shows the evolution during the first 50 days of the isotropic macroscopic strains obtained numerically and analytically for the interface thicknesses of 1,100 and $250 \mu \mathrm{m}$. We observe that the magnitude of these strains is only moderately affected by the ITZ thickness; indeed, the strain difference between the lower and higher thickness value is no more than 5\%. Moreover, the time evolution of the macroscopic strains is also limited to about 3\% at 50 days. The comparison between FE numerical and analytical results shows a good accuracy for $h=1 \mu \mathrm{m}$ (maximum discrepancy of less than 1\% at 50 days), and small differences appear for higher thicknesses (about 2\% at 50 days for $h=250 \mu \mathrm{m}$ ). Fig. 4 (right) presents the evolutions of the average stresses in aggregate and matrix phases obtained numerically and analytically for the 3 considered interface thicknesses. As the volume fraction of the inclusion phase is $50 \%$ and no macroscopic stresses are applied to the specimen, the average stresses in both phases are opposite. We observe that the stresses progressively relax from a tensile and compressive state for the aggregate and matrix, respectively, in agreement with the values of CTE for these two components. As expected since the ITZ mechanical properties are lower than the ones of the matrix and aggregates, increasing the ITZ thickness tends to lessen the magnitude of the average stresses. We note that the model provides relatively correct estimations of these quantities, with a trend to underestimate their magnitude.

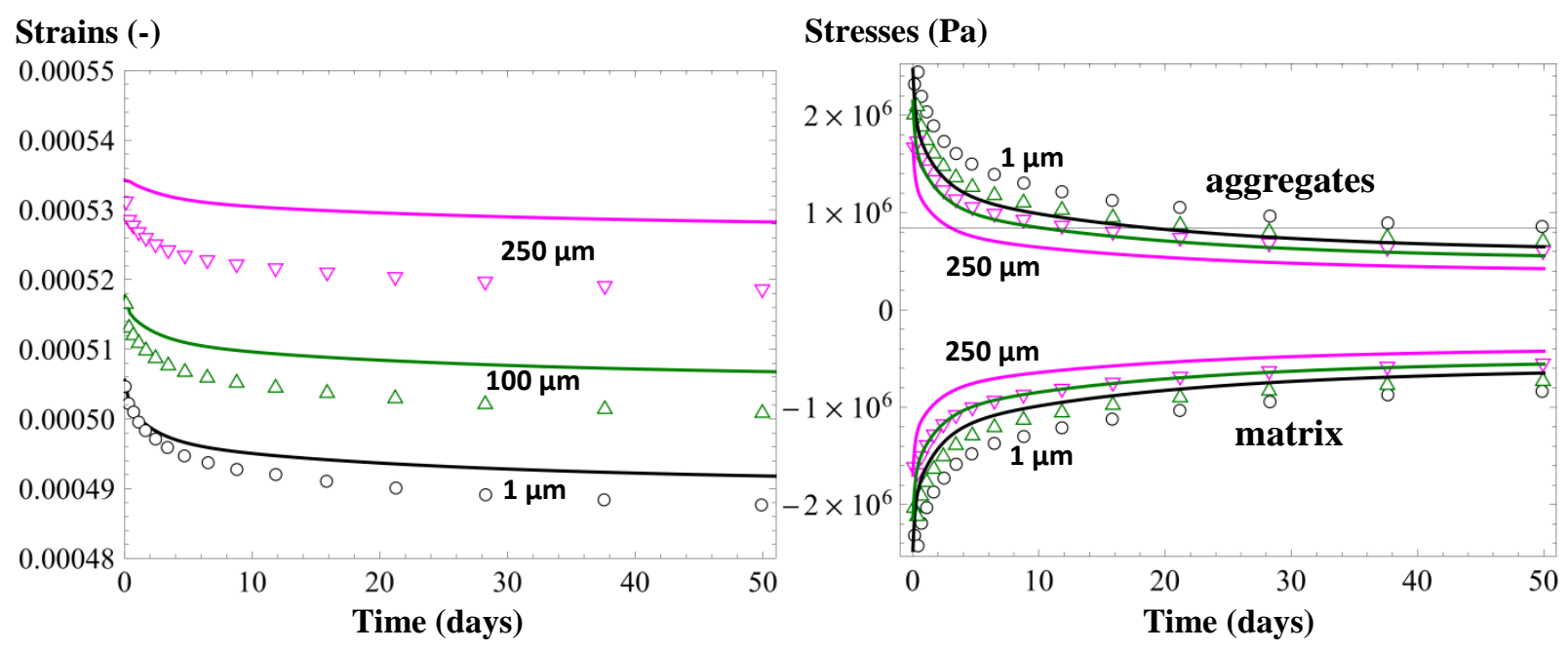

Fig. 4. Macroscopic strains due to a temperature increase of $40^{\circ} \mathrm{C}$ obtained numerically (symbols) and analytically (lines) for interface thicknesses of 1, 100 and $250 \mu \mathrm{m}$ (left). Corresponding average stresses in the matrix and aggregate phase for the same interface thicknesses (right).

\section{Conclusion}

We have presented results regarding the modelling and FE simulation of the thermoviscoelastic response of cementitious materials with a special regard to the effects of ITZ. We consider from the numerical-analytical confrontation that the model developed is globally able to correctly reproduce the main features of the material behavior. The observed discrepancies may be attributed partly to the fact that the analytical estimations are obtained with spherical aggregates, while the numerical samples are generated with polyhedrons. Overall, we have observed that the effects of ITZ are nonsurprisingly small for thicknesses lower than about $25 \mu \mathrm{m}$, i.e. for concrete, while they have significantly more impact for higher values corresponding to mortars.

The next development step of the FE model concerns the introduction and modelling of microcracking in both ITZ and matrix phase, which will permit to more precisely reproduce the material behavior in diverse situations and loading scenarios, including creep in compression and tension. 


\section{Acknowledgments}

Financial supports from EDF via MACENA project and Matix project from CEA are gratefully acknowledged.

\section{References}

[1] M.P. Lutz, P.J.. Monteiro, R.W. Zimmerman, Inhomogeneous interfacial transition zone model for the bulk modulus of mortar, Cement and Concrete Research. 27 (1997) 1113-1122.

[2] K.L. Scrivener, A.K. Crumbie, P. Laugesen, The interfacial transition zone (ITZ) between cement paste and aggregate in concrete, Interface Science. 12 (2004) 411-421.

[3] H.L. Duan, X. Yi, Z.P. Huang, J. Wang, A unified scheme for prediction of effective moduli of multiphase composites with interface effects: Part II-Application and scaling laws, Mechanics of Materials. 39 (2007) 94-103. doi:10.1016/j.mechmat.2006.02.010.

[4] R.M. Christensen, L.B. Freund, Theory of viscoelasticity, Journal of Applied Mechanics. 38 (1971) 720.

[5] M.E. Gurtin, E. Sternberg, On the linear theory of viscoelasticity, Archive for Rational Mechanics and Analysis. 11 (1962) 291-356.

[6] M.A. Biot, Theory of deformation of a porous viscoelastic anisotropic solid, Journal of Applied Physics. 27 (1956) 459-467.

[7] Z. Hashin, Viscoelastic behavior of heterogeneous media, Journal of Applied Mechanics. 32 (1965) 630.

[8] J. Mandel, Cours de mécanique des milieux continus, Gauthier-Villars, 1966.

[9] M.-Q. Thai, B. Bary, Q.-C. He, A homogenization-enriched viscodamage model for cementbased material creep, Engineering Fracture Mechanics. 126 (2014) 54-72.

[10] Z. Hashin, Thermoelastic properties of particulate composites with imperfect interface, Journal of the Mechanics and Physics of Solids. 39 (1991) 745-762.

[11] R. Christensen, K. Lo, Solutions for effective shear properties in three phase sphere and cylinder models, Journal of the Mechanics and Physics of Solids. 27 (1979) 315-330.

[12] H.L. Duan, X. Yi, Z.P. Huang, J. Wang, A unified scheme for prediction of effective moduli of multiphase composites with interface effects. Part I: Theoretical framework, Mechanics of Materials. 39 (2007) 81-93. doi:10.1016/j.mechmat.2006.02.009.

[13] C. Bourcier, W. Dridi, L. Chomat, E. Laucoin, B. Bary, E. Adam, Combs: open source python library for RVE generation. Application to microscale diffusion simulations in cementitious materials, in: Paris, 2013.

[14] T. de Larrard, B. Bary, E. Adam, F. Kloss, Influence of aggregate shapes on drying and carbonation phenomena in 3D concrete numerical samples, Computational Materials Science. 72 (2013) 1-14.

[15] B. Bary, C. Bourcier, T. Helfer, Numerical analysis of concrete creep on mesoscopic 3D specimens, in: Mechanics and Physics of Creep, Shrinkage, and Durability of Concrete and Concrete Structures, Vienne, Austria, 2015.

[16] Z.P. Bazant, G. Cusatis, L. Cedolin, Temperature effect on concrete creep modeled by microprestress-solidification theory, J. Eng. Mech. 130 (2004) 691-699.

[17] W. Ladaoui, T. Vidal, A. Sellier, X. Bourbon, Effect of a temperature change from 20 to $50^{\circ} \mathrm{C}$ on the basic creep of HPC and HPFRC, Materials and Structures. 44 (2011) 1629-1639.

[18] C.R. Cruz, M. Gillen, Thermal expansion of Portland cement paste, mortar and concrete at high temperatures, Fire Mater. 4 (1980) 66-70. doi:10.1002/fam.810040203.

[19] M. Wyrzykowski, P. Lura, Controlling the coefficient of thermal expansion of cementitious materials - A new application for superabsorbent polymers, Cement and Concrete Composites. 35 (2013) 49-58. doi:10.1016/j.cemconcomp.2012.08.010.

[20] H.L. Duan, B.L. Karihaloo, Thermo-elastic properties of heterogeneous materials with imperfect interfaces: Generalized Levin's formula and Hill's connections, Journal of the Mechanics and Physics of Solids. 55 (2007) 1036-1052. doi:10.1016/j.jmps.2006.10.006. 
[21] T. Helfer, B. Michel, J.-M. Proix, M. Salvo, J. Sercombe, M. Casella, Introducing the opensource mfront code generator: Application to mechanical behaviours and material knowledge management within the PLEIADES fuel element modelling platform, Computers \& Mathematics with Applications. 70 (2015) 994-1023. 